\title{
From Small Coronary Artery Aneurysm to Giant Left Ventricle Aneurysm
}

\author{
Sergio Sciacca ${ }^{a}$ Giuseppe Maria Raffa ${ }^{a}$ Giovanni Gentile ${ }^{b}$ Michele Pilato ${ }^{a}$ \\ ${ }^{a}$ Cardiac Surgery and Heart Transplantation Unit, Department for the Treatment and Study of Cardiothoracic Diseases \\ and Cardiothoracic Transplantation, and ${ }^{b}$ Radiology Service, Department of Diagnostic and Therapeutic Services, \\ IRCCS-ISMETT (Mediterranean Institute for Transplantation and Advanced Specialized Therapies), Palermo, Italy
}

\section{Key Words}

Coronary artery $\cdot$ Aneurysm $\cdot$ Cardiac surgery

\begin{abstract}
Objective: To underscore how challenging the treatment of a coronary artery aneurysm (CAA) can be and highlight the need for consensus guidelines based on focused registries. Clinical Presentation and Intervention: A 58-year-old man presented with acute coronary syndrome and underwent elective stent placement on a right CAA. The procedure was complicated by inferior acute myocardial infarction; 8 months later, due to remodeling toward a left ventricular aneurysm of the inferior wall, he experienced several episodes of sustained ventricular tachycardia that required urgent surgical treatment. Conclusion: The best therapeutic option for CAA is still a matter of controversy, and though the percutaneous approach can meet the technical challenges, 'heart team'-based decision-making is recommended. The case reported here showed that percutaneous treatment of CAA can be a challenging procedure, even in experienced and high-volume centers. In our patient's case, undersizing the covered stent led to further migration and to a complication that was more severe than the original disease.
\end{abstract}

(c) 2016 S. Karger AG, Basel

\section{KARGER}

E-Mail karger@karger.com www.karger.com/mpp

\section{(c) 2016 S. Karger AG, Basel}

This is an Open Access article licensed under the terms of the Creative Commons Attribution-NonCommercial 3.0 Unported license (CC BY-NC) (www.karger.com/OA-license), applicable to the online version of the article only. Distribution permitted for non-commercial purposes only.

\section{Introduction}

The incidence of coronary artery aneurysm (CAA) diagnosed by angiography varies from 1.5 to $5 \%$ [1-3]. The most common etiology is atherosclerosis, accounting for $50 \%$ of coronary aneurysms diagnosed in adults, followed by Kawasaki disease, congenital aneurysms, arteritis, mycotics and connective tissue disorders $[1,2]$.

Stent placement has been reported as an emerging cause of iatrogenic CAA [2]. The complications of CAA include thrombosis, distal embolization, rupture and coronary spasms $[1,4]$ and therefore treatment is sometimes indicated. Hence, we report a case of a CAA treated with stent placement that further required an urgent cardiac operation.

\section{Case Report}

A 58-year-old male presented with chest pain at another institution where he had been diagnosed with acute coronary syndrome. He underwent coronary angiography and bare-metal stent placement in the proximal left anterior descending (LAD) artery in May 2012.

During the same procedure, a right $\mathrm{CAA}$ of $2 \mathrm{~cm}$ was diagnosed and after 6 months, a second elective percutaneous procedure with

Giuseppe Raffa, MD

Istituto Mediterraneo per i Trapianti e Terapie ad alta specializzazione Via Tricomi 5

IT-90127 Palermo (Italy)

E-Mail giuseppe.raffa78@gmail.com 


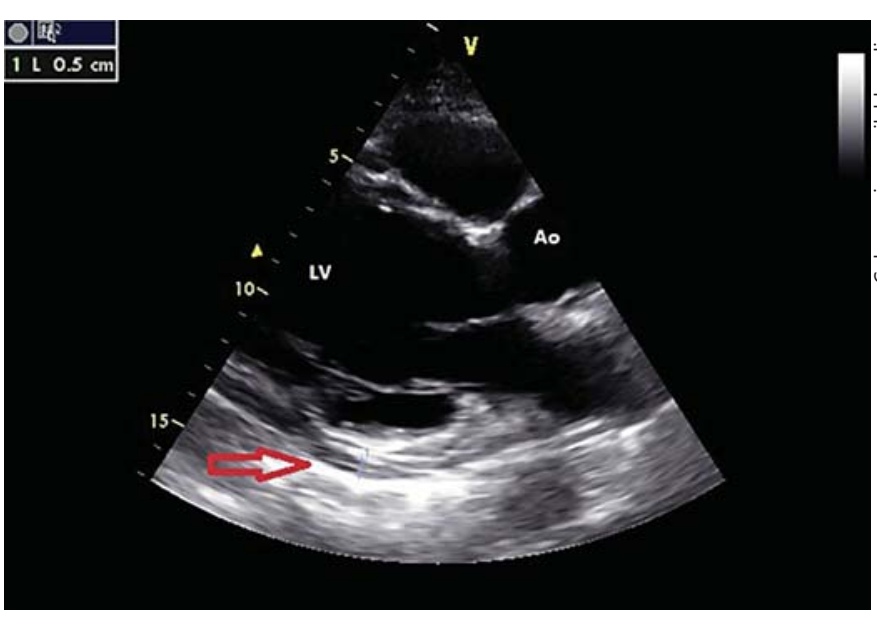

Fig. 1. Preoperative long-axis transthoracic echocardiogram shows the LV inferior aneurysm (arrow) and pericardial effusion.

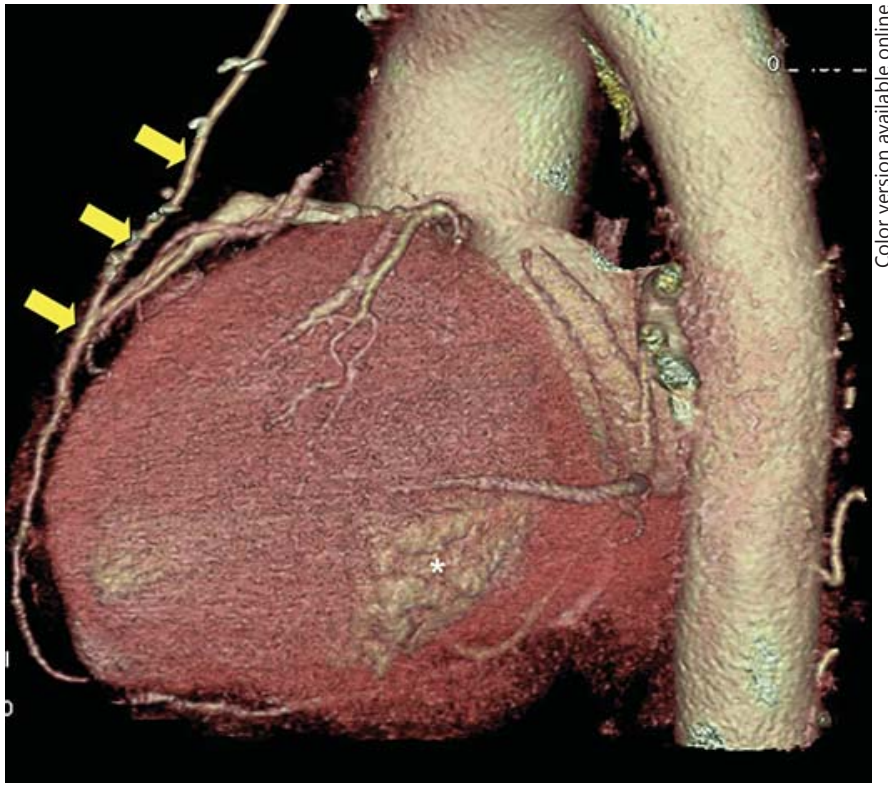

Fig. 3. Follow-up cardiac CT scan findings: control after aneurysm repair (asterisk) and LIMA to LAD bypass (arrows).
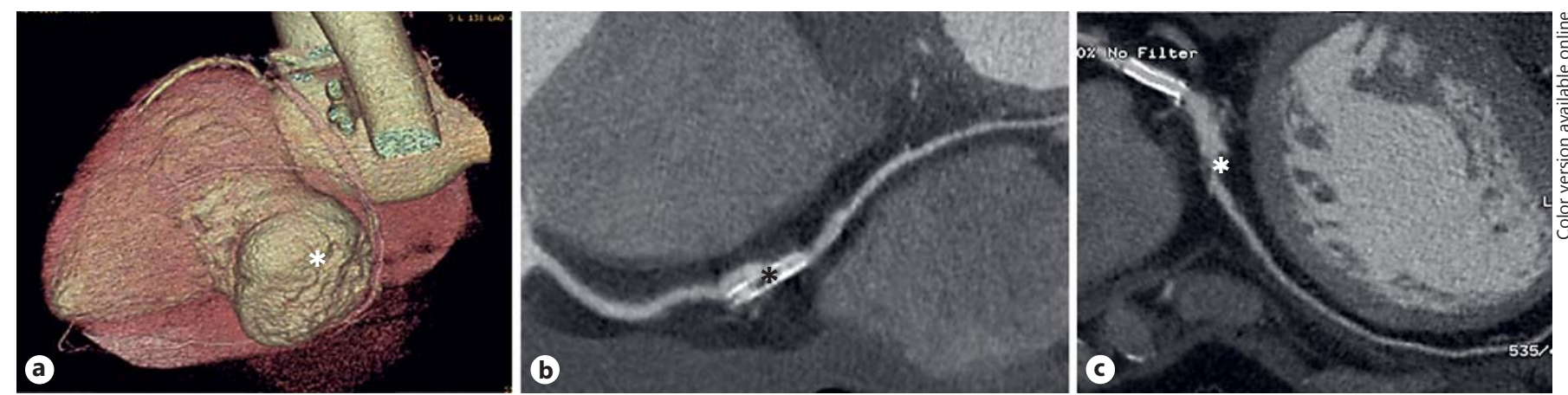

Fig. 2. Preoperative cardiac CT scan findings. a Aneurysm of the LV inferior wall (asterisk) after iatrogenic myocardial ischemia. b Right CAA with dislocated stent. The asterisk indicates intrastent occlusive stenosis. c Patency of stent in proximal LAD and significant stenosis (asterisk) of the middle tract.

a stent was performed to exclude the CAA. The procedure was complicated $24 \mathrm{~h}$ later by inferior acute myocardial infarction despite an attempt at percutaneous revascularization, which unfortunately failed.

Eight months later, the patient was readmitted for an episode of ventricular tachycardia. On admission, a transthoracic echocardiogram showed a left ventricular (LV) aneurysm of the inferior wall (fig. 1), pericardial effusion and an ejection fraction of $40 \%$. The patient was then transferred to our Cardiac Surgery Unit. A cardiac computed tomography (CT) scan showed a giant LV aneurysm involving the inferior wall (fig. 2a), right coronary artery occlusion due to displacement of the proximal tip of the stent (fig. 2b) and an LAD intrastent stenosis (fig. 2c).

The patient underwent surgical LV restoration using the Dor procedure as well as arterial coronary artery bypass graft on the LAD artery. The postoperative course was uncomplicated, and the patient was discharged home 6 days later, with an ejection fraction (EF) of $50 \%$. The CT scan at the 6-month follow-up showed good surgical results (fig. 3). 


\section{Discussion}

In this case, an elective, low-risk procedure produced a severe complication. Its surgical correction, despite a higher operative risk led to a favorable LV remodeling and better long-term results.

The pathogenesis of CAA is not fully understood. Inflammation, as a result of a systemic or local process, leads to the upregulation of proinflammatory cytokines and enzymes and the downregulation of protective mediators [3]. The effect of these is either an initial direct degrading of the tunica media or a later degradation secondary to tunica intima involvement, as in arterosclerotic aneurysm, and can sometimes be present in all components of the arterial wall matrix, including such proteins as elastin, collagen, proteoglycans, laminin and fibronectin. Thinning of the arterial wall, increased wall stress and dilatation seem to complete the process of CAA formation [3]. Our case underscored how CAA treatment was a challenging procedure even for experienced physicians, with such documented complications as side-branch closure, thrombosis and intrastent stenosis $[5,6]$.

Surgical treatment of CAAs, i.e. aneurysm ligation, placation or resection with or without revascularization, has a $1-2 \%$ operative risk comparable to coronary artery procedures $[1-3,7]$. Percutaneous treatment of CAA to exclude CAAs with a bare metal stent, a covered stent or coil embolization has been suggested as a safe procedure in several case reports [1-4].

\section{Conclusion}

The case reported showed that percutaneous treatment of CAA was a challenging procedure, even in experienced and high-volume centers. In our patient's case, undersizing the covered stent led to further migration and to a complication that was more severe than the original disease. While surgical treatment of CAAs is a valid option, focused studies on a large number of patients are needed to determine the best strategy. We believe that, in the meantime, careful evaluation by a 'heart team' is advisable for the management of this increasing population of patients.

\section{Disclosure Statement}

There were no conflicts of interest.

\section{References}

CAA Complications
1 Syed M, Lesch M: Coronary artery aneurysm: a review. Prog Cardiovasc Dis 1997;40:77-84.

2 Zeb M, McKenzie DB, Scott PA, et al: Treatment of coronary aneurysms with covered stents: a review with illustrated case. J Invasive Cardiol 2012;24:465-469.

3 Mata KM, Fernandes CR, Floriano EM, et al: Coronary artery aneurysms: an update; in Lakshmanadoss U (ed): Novel Strategies in Ischemic Heart Disease, chapt 21, 2012. http://www.intechopen.com/books/novelstrategies-in-ischemic-heart-disease.

4 Crimi G, Belotti S, Iannone A, et al: Sequencial coronary angiograms unveil the progres- sion of an acquired coronary aneurysm. Eur Heart J 2013;34:2924.

5 Bajaj S, Parikh R, Hamdan A, et al: Coveredstent treatment of coronary aneurysm after drug-eluting stent placement: case report and literature review. Tex Heart Inst J 2010;37: 449-454.

6 Terasawa A, Yokoi T, Kondo K: Stent-assisted coil embolization of coronary artery aneurysm. J Invasive Cardiol 2013;25:E175E177.

7 Harandi S, Johnston SB, Wood RE, et al: Operative therapy of coronary arterial aneurysm. Am J Cardiol 1999;83:1290-1293. 